\title{
Metodologias ativas como estratégia pedagógica para promoção do ensino-aprendizagem em Odontologia: relato de experiência
}

\author{
Soraya de Azambuja Berti Couto*; Paulo Henrique Couto Souza** \\ * Doutora em Estomatologia, Professora Adjunta, Disciplina de \\ Estomatologia, Curso de Odontologia, Escola de Ciências da Vida, \\ Pontifícia Universidade Católica do Paraná \\ ** Doutor em Cirurgia Bucomaxilofacial, Professor Titular, \\ Disciplina de Estomatologia, Curso de Odontologia, Escola de \\ Ciências da Vida, Pontifícia Universidade Católica do Paraná
}

Recebido em 10/04/2019. Aprovado em 06/06/2019.

\begin{abstract}
RESUMO
Ao longo da história, o ensino e o aprendizado nos Cursos de Odontologia no Brasil vem sendo exercidos com ênfase no desenvolvimento de conhecimentos científicos e habilidades técnicas que são indispensáveis para a profissão. Porém, considerando-se as atuais necessidades apresentadas pela sociedade moderna, a importância em propiciar ao estudante de graduação em Odontologia uma formação integral, holística e baseada em competências, que vão além do tecnicismo, torna-se emergente. O presente artigo tem como objetivo relatar a experiência da utilização de metodologias ativas, com atividades lúdicas, como estratégia pedagógica para a promoção do ensino-aprendizagem, baseada em competências, na disciplina de Estomatologia, do Curso de Odontologia, da Pontifícia Universidade Católica do Paraná. De acordo com os relatos apresentados pelos estudantes desta disciplina, conclui-se que as metodologias ativas podem ser utilizadas como estratégia pedagógica complementar para o ensino e aprendizagem em Odontologia.
\end{abstract}

Descritores: Ensino. Aprendizado. Odontologia.

\section{INTRODUÇÃO}

No Brasil, o processo de ensino e aprendizagem é baseado, tradicionalmente, na teoria da transmissibilidade, ou seja, na transmissão de informações. Também conhecido como ensino tradicional, essa proposta de aprendizagem é baseada "no transmitir", utilizando-se, para tanto, de aulas expositivas, nas quais os estudantes aprendem de forma passiva as informações transmitidas pelo 
professor $^{1,2}$. Entretanto, o contexto da Educação no país, com destaque para o ensino superior, vem enfrentando sérias e profundas dificuldades para acompanhar as mudanças ocorridas na sociedade ao longo dos anos. Atualmente "o aprender" pode acontecer de diferentes maneiras como pelo estudo individual, em grupos, por meio de intercâmbios, entre outros ${ }^{3}$. Araújo e Mazur $(2013)^{2}$ destacaram que o ensino tradicional está relacionado à problemas como a evasão escolar, a aprendizagem mecanizada e a desmotivação apresentada pelos estudantes de nível médio e de disciplinas básicas de ensino superior.

Paralelamente, nos últimos anos, observase uma atenção ascendente em relação a novas estratégias pedagógicas na promoção do ensino e aprendizagem, como por exemplo, as metodologias ativas. Trata-se de uma busca por métodos de ensino que propiciem um maior engajamento por parte dos docentes e estudantes, favorecendo $\mathrm{o}$ processo de ensino $\mathrm{e}$ aprendizagem. Esta, pode ser definida como qualquer método instrucional que envolve os estudantes, de maneira ativa, no processo de aprendizagem $^{4}$. Consequentemente, para que o aprendizado significativo seja alcançado pelo estudante, há necessidade de que os mesmos desenvolvam competências ${ }^{5}$ e reflitam sobre o que estão fazendo, mostrando-se ativos neste processo. Spricigo $(2014)^{6}$ destacou que situações de contexto real aproximam o estudante da aplicação prática do conhecimento, promovendo a aprendizagem, desenvolvendo competências e habilidades relacionadas à resolução de problemas reais, favorecendo a tomada de decisão, a capacidade de argumentação e ao trabalho efetivo em equipe. Por outro lado, o aprendizado ativo, muitas vezes é entendido como um "modismo educacional" por alguns grupos de docentes e estudantes o que suscita dúvidas sobre o que realmente é a aprendizagem ativa ${ }^{4}$.

Diante desta nova realidade, algumas instituições de educação vêm adotando as metodologias ativas como uma nova forma de promover o ensino e a aprendizagem ${ }^{7}$, inclusive capacitando o corpo docente e, conjuntamente, preparando os discentes para tal. Embora, no Brasil, este assunto esteja sendo muito discutido atualmente, é importante salientar que, a Universidade de Brasília pode ter sido uma das primeiras Universidade do mundo a aplicar uma abordagem de ensino e aprendizagem diferenciada, por meio do método PBL (aprendizagem baseada em problemas). A Universidade de Brasília foi fundada em 1960 e começou a lecionar em 1962 e dentre suas características principais destacavam-se a solução de problemas, a interdisciplinaridade, a experimentação, a integração de pesquisa e ensino e a colaboração com a sociedade ${ }^{8}$.

Tradicionalmente, a educação odontológica formal no Brasil, iniciou-se junto à Medicina, em 1879 com a introdução da denominada disciplina de "Cirurgia Dental", inicialmente lecionada nas Escolas de Medicina. O então Imperador do Brasil, Dom Pedro II, formalizou por decreto a Odontologia como profissão em 1884, e os primeiros "Cursos de Odontologia" foram criados nas Escolas de Medicina no Rio de Janeiro e na Bahia. Paralelamente em 1900, a Odontologia também foi estabelecida como uma profissão na Europa e nos Estados Unidos ${ }^{9}$. Daí por diante, em 1911, a disciplina de "Técnica Dental" foi introduzida no Brasil, possibilitando o treinamento em manequins, o que representou um avanço nos aspectos técnicos de educação odontológica, que perdurariam até os dias atuais. Já em 1919, os Cursos de Odontologia no Brasil passaram a ser desenvolvidos em quatro anos com ênfase nos conhecimentos em Biologia. A partir de 1933, as então Escolas de Odontologia tornaram-se 
autônomas, iniciando a era dos cursos de graduação e também de especialização. Atualmente, a Odontologia no Brasil ainda está evoluindo, como resultado da Lei de Diretrizes e Bases de 1996 e das Diretrizes Curriculares Nacionais de $2002^{10,11}$.

Os cursos de Odontologia do Brasil, assim como outros cursos de ensino superior, vêm buscando evoluir pedagogicamente diante às novas formas de ensino e aprendizagem e, para tanto, reestruturam suas matrizes curriculares favorecendo ainda mais o emprego de metodologias ativas. Entretanto, a Odontologia do Brasil ainda é desenvolvida enfatizando o tecnicismo necessário ao pleno desenvolvimento das habilidades profissionais de um cirurgiãodentista $^{12}$. De fato, o desenvolvimento de habilidades técnicas é uma característica essencial para a formação do futuro cirurgiãodentista, sem as quais, os profissionais não poderiam exercer a Odontologia intervencionista e terapêutica. Neste sentido, a pedagogia do condicionamento e tecnicista baseada na teoria de aprendizagem Behaviorista, que busca mudanças comportamentais do estudante ${ }^{13}$, ainda é muito utilizada para o desenvolvimento de habilidades técnicas em Odontologia, o que se torna indispensável. Porém, paralelamente a esta característica própria dos cursos de graduação em Odontologia, os mesmos também apresentam a necessidade emergente de se adaptar às novas metodologias de ensino, buscando cada vez mais integrar a teoria e a prática, o ensino/serviço e o desenvolvimento de competências. $O$ que se questiona é: até que ponto o processo de ensino e aprendizado da Odontologia, estruturado predominantemente no tecnicismo, é suficiente para formar profissionais que atendam e resolvam as demandas da sociedade que está cada vez mais informada e com necessidades mais abrangentes?
Diante deste contexto, o presente artigo tem como objetivo relatar a experiência da utilização de metodologias ativas, com atividades lúdicas, como estratégia pedagógica para a promoção do ensino-aprendizagem, na Disciplina de Estomatologia, do Curso de Odontologia, da Pontifícia Universidade Católica o Paraná (PUCPR).

\section{RELATO DE EXPERIÊNCIA}

O presente relato de experiência foi resultado da participação em um projeto de formação docente, conduzido pelo CrEARE (Centro de Ensino e Aprendizagem) da PUCPR, o qual foi promovido e fomentado pela Agência Financiadora de Inovação e Pesquisa (FINEP), entre 2017 e 2019.

Este relato de experiência é de caráter descritivo e apresenta como cenário de experiência a Disciplina de Estomatologia, do Curso de Graduação em Odontologia da PUCPR, que ocorreu durante o primeiro e segundo semestres de 2018. A referida Disciplina é teórico-prática, ministrada no $7^{\circ}$ período do Curso de Odontologia e apresenta carga horária de 72 horas. Em média, tem cerca de 45 estudantes matriculados por semestre e, atualmente, recebe também estudantes intercambistas da Colômbia, Bolívia, Chile e Bélgica. A parte teórica (2 aulas) é desenvolvida em sala de aula e a parte prática (2 aulas) corresponde ao atendimento de pacientes na Clínica de Odontologia da PUCPR. Ao longo dos dois semestres de aplicação das metodologias ativas, os estudantes realizaram as atividades em grupos (Team Based Learning - TBL), previamente organizados pelo professor, a partir de um questionário estruturado respondido pelos mesmos ao início do semestre, buscando integrar modelos mentais diferentes que favorecessem o processo de ensino e aprendizagem ${ }^{14}$. 
A seguir serão relatadas algumas experiências vividas pelo professor ao longo do primeiro e segundo semestres letivos de 2018, utilizando-se para tanto, formas lúdicas de apresentar e trabalhar junto aos estudantes com os temas de estudo propostos, com o objetivo de desenvolver os resultados de aprendizagem esperados pela disciplina.

\section{Atividade primeiro dia de aula}

O professor iniciou a primeira aula do semestre projetando uma imagem de alpinistas no começo da escalada de uma montanha, traçando um paralelo com a Disciplina, buscando a sensibilização do estudante e enfatizando que para se chegar ao objetivo final (no caso, o topo da montanha), seria importante o trabalho integrado entre estudantes e professores. Além disso, o professor dialoga com o estudante com o objetivo de mostrar que a Odontologia vai muito mais além do que doenças como cárie e periodontite. Atualmente, o cirurgião-dentista, possui uma grande responsabilidade como profissional de saúde em identificar alterações bucais e/ou sistêmicas como o câncer, AIDS, doenças autoimunes, diabetes, doenças sexualmente transmissíveis como HPV, sífilis e, até mesmo, pacientes que apresentem maior risco ao desenvolvimento de doenças cardiovasculares. Os efeitos adversos do uso de medicações sistêmicas na saúde bucal, também se destacam neste contexto. Como consequência, conscientizar o estudante do Curso de Graduação em Odontologia de que o paciente não é apenas um "procedimento" e que habilidade manual, apesar de ter fundamental importância na formação do cirurgião-dentista, por si só, não é suficiente para o cuidado do paciente de forma integral e humanizada.

Após esta sensibilização preliminar, procedeu-se a apresentação do professor, mostrando imagens da atividade profissional, desde a época de formação acadêmica até os dias atuais, o que causou um impacto positivo aos estudantes neste momento inicial. Em seguida, solicitou-se que os estudantes se organizassem em grupos para a atividade de interação. Nesta atividade, os estudantes foram desafiados a montar uma imagem, previamente fornecida pelo professor, a partir de 7 peças do quebra-cabeças chinês Tangram. A equipe que resolvesse o desafio de maneira mais rápida receberia um "prêmio" como reconhecimento. O resultado foi melhor do que o esperado, os estudantes deram risadas, brincaram, gritaram, o que propiciou maior integração entre eles mesmos. Os próprios estudantes intercambistas se motivaram a participar das equipes contribuindo com suas opiniões e experiências oriundas dos seus locais de origem. A partir deste jogo, e do resultado que as equipes foram apresentando, contextualizouse a disciplina, sua aplicação prática diante ao mercado de trabalho, além de destacar a importância do trabalho em grupo, propiciando o desenvolvimento da competência em trabalhar de forma interdisciplinar ${ }^{5}$.

O resultado inicialmente apresentado, utilizando-se esta metodologia no primeiro contato entre o professor e os estudantes, em cada um dos semestres de aplicação, foi a boa receptividade dos estudantes e o engajamento com o qual assumiram e realizaram a atividade proposta. Além disso, ao final da aula, o professor foi abordado por um estudante após todos os outros saírem da sala. Este estudante disse:

"Professora que aula foi esta? Muitos dos meus colegas não têm a consciência de que é muito importante dar um retorno ao professor sobre as atividades desenvolvidas. Mas eu tenho professora, e por isso estou aqui para te dizer que eu nunca tive uma aula tão boa e tão estimulante. É claro que tenho muita 
afinidade com este tema, mas fiquei muito motivado e entusiasmado para o semestre na Estomatologia, me despertando ainda mais a vontade de seguir esta área. Por isso professora, eu me coloco à disposição para fazer qualquer tipo de trabalho da disciplina: apresentação de trabalho, estágio, caso clínico, ajudar vocês professores em qualquer coisa". Estudante 1

\section{Atividade de estudo de caso}

Outra atividade programada nesta disciplina foi um estudo de caso, elaborado previamente pelo professor. Esta atividade teve por objetivo alcançar o segundo resultado de aprendizagem (RA 2) previsto na referida Disciplina: Elaborar o diagnóstico clínico de alterações bucais patológicas a partir de dados do exame clínico extrabucal e intrabucal, considerando o suporte de exames complementares.

Entretanto, para a realização satisfatória desta atividade, os estudantes necessitaram aplicar os conhecimentos obtidos anteriormente com o desenvolvimento do primeiro resultado de aprendizagem (RA 1) alcançado: Analisar alterações bucais e o impacto de fatores do contexto social, psicológico e de saúde do paciente na saúde bucal, identificando variantes anatômicas, lesões patológicas e o risco de repercussões sistêmicas.

Esta atividade de estudo de caso compreendeu 2 semanas de aulas teóricas, e a avaliação realizada pelo professor foi formativa, com feedback imediato aos estudantes, no primeiro dia nos pequenos grupos e no segundo dia para o grande grupo, o qual incluiu toda a turma de estudantes.

Este estudo de caso foi realizado em duas etapas, pelos estudantes, nos pequenos grupos previamente organizados ao início do semestre, ao longo das aulas teóricas desenvolvidas em duas semanas. Na primeira semana, os estudantes estudaram e discutiram um caso clínico de um paciente com queixa de xerostomia, buscando estabelecer uma linha de raciocínio, correlacionando as informações apresentadas pela anamnese e exame físico, extra e intrabucais. Ao final do primeiro dia de aula, cada pequeno grupo de estudantes elaborou um mapa mental mostrando esta correlação e desenvolvendo a competência do pensamento crítico (figura 1) interdisciplinar ${ }^{5}$. Ao longo da aula, o professor pôde auxiliar os pequenos grupos, realizando feedbacks imediatos aos mesmos, a partir do que cada grupo estava discutindo e produzindo.

No início do segundo dia da atividade de estudo de caso, o professor pediu que um relator de cada grupo, escolhido aleatoriamente, explicasse brevemente aos demais grupos da sala a linha de raciocínio utilizada pelo grupo, procedendo então, a avaliação formativa desta primeira etapa da atividade. Durante a explanação de cada um dos grupos, o professor realizava os feedbacks imediatos a todos os estudantes, salientando e fundamentando os aspectos principais destacados por cada um dos grupos. Além disso, o professor propiciava a discussão entre os grupos, os quais chegaram ao objetivo final da atividade, porém destacando diferentes formas de condução do referido caso.

Ao final de todas as apresentações, o professor realizou uma fundamentação teórica destacando e apresentando o raciocínio clínico para a condução do referido caso, que, apesar de integrar diferentes temas de estudo, apresentava como principal tema a hipossalivação. No último slide de explanação, o professor finalizou a aula propondo uma dinâmica/vivência, que ilustrasse todas as orientações que deveriam ser dadas aos pacientes diante à casos clínicos que envolvessem este tema de estudo, convidando os 
estudantes a fazerem degustação de estimulantes de fluxo salivar como limão e balas cítricas, além da saliva artificial, desconhecida pela grande maioria dos estudantes. Nesta oportunidade os estudantes deram alguns depoimentos sobre como foi o desenvolvimento desta atividade.

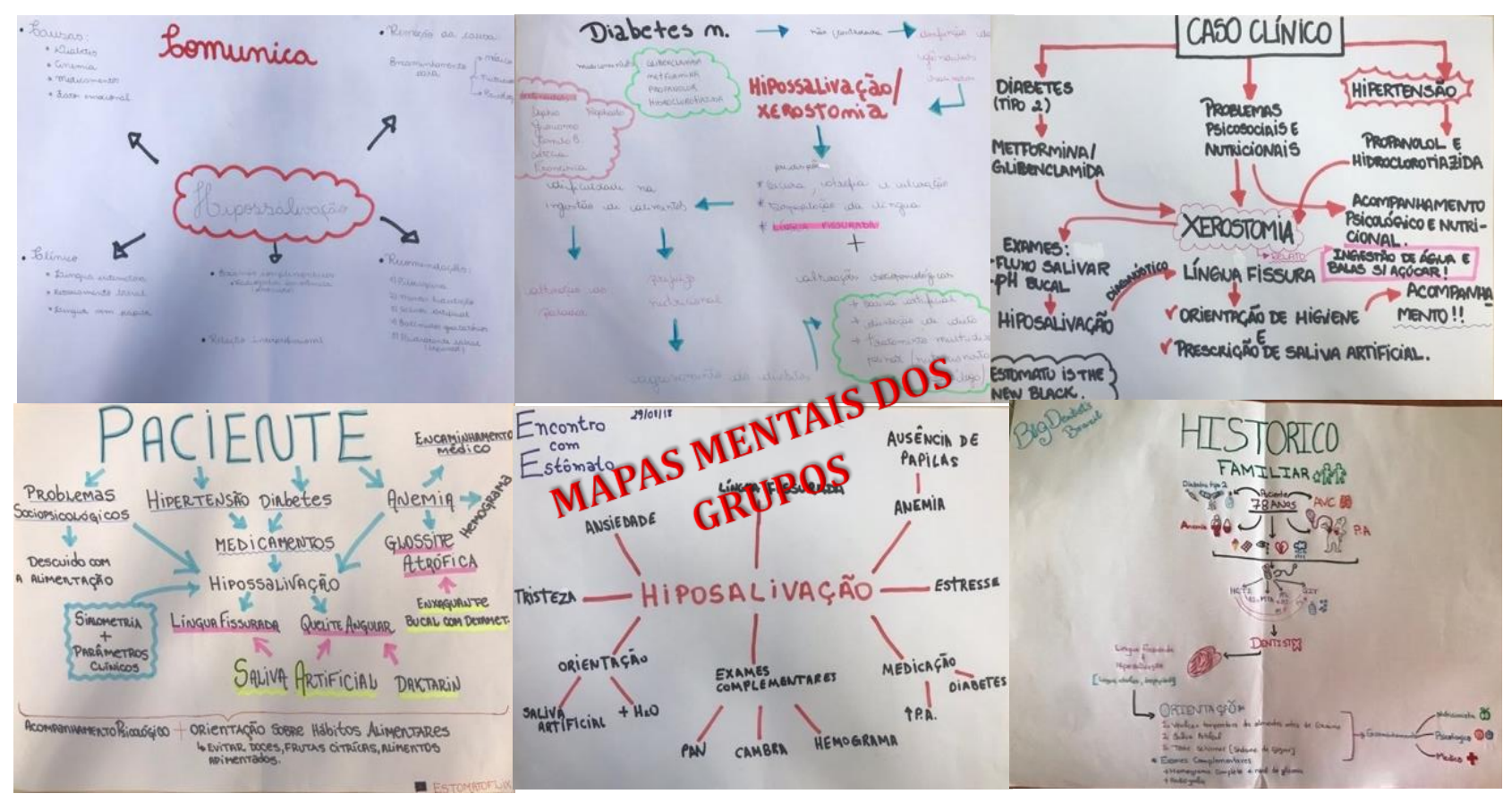

Figura 1. Exemplos de mapas mentais produzidos pelos pequenos grupos da Disciplina de Estomatologia

"Adorei a atividade, aprendi muito mais do que uma aula expositiva comum. No início da atividade senti um pouco de dificuldade por não saber como começála, porém, com o desenvolver do caso fui relembrando muitas coisas. Além disso a finalização da atividade concluiu perfeitamente o caso clínico." Estudante 2 "A atividade realizada em aula foi muito interessante e a forma como ela foi realizada nos estimulou a pesquisar artigos e a aprender mais do que uma aula recheada de slides. Foram aulas dinâmicas e muito produtivas." Estudante 3

"Achei que a aula foi muito produtiva desde o momento que foi proposto fazer a pesquisa acerca do tema do caso clínico $e$, após, montar um fluxograma. Acredito que este tipo de aula/atividade consegue atender a necessidade de conhecimento que nós alunos precisamos ter, principalmente na atuação clínica. Parabéns Professora!"

Estudante 4

"A atividade foi bem produtiva e com excelente aplicação da metodologia ativa. Parabéns!"

Estudante 5

"Eu achei toda a atividade maravilhosa, na verdade achava que não ia aprender muito, sempre falava da diabetes, da 
anemia, mais nunca entendi realmente como os medicamentos podiam afetar o quadro clínico. Hoje, pela primeira vez, não só tive um conhecimento do tema, sinto que compreendi e sei que não esquecerei porque aprendi com a vivência e não com a memória. $O$ que mais amei foi experimentar a saliva artificial. Eu achava que era diferente, muito obrigada pela oportunidade de aprender através da experiência. Amei a aula!"

Estudante Intercambista (Colômbia)

"Estou fascinada com a aula, gostei muito da atividade e também da sequência da aula... Creio que é uma maneira de compartilhar conhecimentos em grupo e aprender sob diferentes pontos de vista... $O$ caso clínico apresentado foi muito interessante, aprendi a manejar e conduzir o paciente da melhor maneira possivel como futura dentista. Parabéns professora por todas as aulas e pela dedicação e empenho." Estudante Intercambista (Peru)

\section{Atividade lúdica de caça-palavras (Flipped Classrom)}

Esta atividade foi proposta com o intuito de aplicar os conhecimentos que foram previamente obtidos pelos estudantes com a atividade preparatória, realizada em ambiente extraclasse. A mesma consistiu no estudo individual de um capítulo de livro indicado no encontro anterior pela professora, tendo como objetivo principal a fundamentação inicial para a aula ministrada presencialmente. Os temas de estudo a serem desenvolvidos com esta atividade estão relacionados ao resultado de aprendizagem 3 (RA 3) da disciplina: Estabelecer condutas de tratamento para alterações bucais patológicas, conforme diagnóstico clínico, de forma individualizada e promovendo a saúde bucal.

Esta atividade lúdica, elaborada pelo professor, foi um caça-palavras, no qual os estudantes, a partir da atividade preparatória, deveriam encontrar as oito principais complicações, extra e intrabucais, relacionadas ao tratamento antineoplásico. A proposta foi de que o grupo que resolvesse esta atividade em um menor tempo receberia um "prêmio" que seria utilizado no decorrer da aula.

A mesma atividade fez com que alguns estudantes que não haviam desenvolvido a atividade preparatória, tivessem dificuldade em resolver o caça-palavras, pois os mesmos não sabiam que palavras procurar. Prevendo uma situação como esta, o professor deixou um material de apoio preparado, o qual foi entregue aos grupos após 8 minutos de início da atividade. Neste momento, os estudantes prontamente começaram a estudar todo o material, até mesmo sem notarem que estavam estudando e discutindo sobre cada grupo de informações para que conseguissem resolver o caça-palavras.

O resultado desta atividade foi muito perceptível, inicialmente por introduzir o tema e valorizar a atividade extraclasse de uma maneira tão "diferente e surpreendente", como os estudantes mesmos relataram ao professor. Após a atividade inicial, o professor realizou a fundamentação teórica da aula, e a atenção e a participação dos estudantes foram muito mais ativas ao longo das discussões dos casos clínicos. Os questionamentos foram muito pertinentes e os objetivos relacionados ao resultado de aprendizagem proposto estavam sendo atingidos. Além disso, o impacto pessoal que foi discutir casos que pareciam improváveis em um contexto real, segundo a própria visão dos estudantes, os fizeram ver e, inclusive, entender a profissão de uma maneira mais abrangente e humanizada, propiciando o cuidado integral e individualizado do ser humano. Mais uma vez as competências 
em saber trabalhar de forma interdisciplinar e em pensar de forma crítica foram desenvolvidas ${ }^{5}$.

Os estudantes realizaram a avaliação de algumas atividades desenvolvidas durante a disciplina, por meio de questionários estruturados, a exemplo das atividades relacionadas ao desenvolvimento do resultado de aprendizagem 3 (RA 3), as quais foram acima descritas. Os estudantes também realizaram a avaliação da disciplina de Estomatologia, após a aplicação de metodologias ativas. A seguir serão apresentados alguns depoimentos realizados pelos estudantes de graduação, os quais foram obtidos por meio dos questionários:

"Acredito que a metodologia ativa contribuiu para a formação do meu conhecimento. Com os meus colegas de grupo pude compartilhar ideias $e$ discussões sobre determinados assuntos. Além disso, as atividades propostas me motivaram a pesquisar mais em casa sobre os temas que a professora propôs" Estudante 6

"Posso expressar que a minha vivência durante as aulas me mostrou como é importante o diálogo entre os alunos e o professor, pois abre uma discussão sobre o tema na qual consegue-se extrair mais informações e assim adquirir mais aprendizado. Junto com a metodologia ativa foram feitos grupos de alunos para, assim, poder ter interação e visões diferentes sobre o mesmo tema, no final havendo um maior raciocínio em torno do assunto, porém todas as discussões e debates realizados eram previamente explicados, tendo assim um direcionamento correto. Eu acho que esse método utilizado para ministrar aulas ajuda o aluno a buscar mais conhecimento $e$ não apenas ficar recebendo aulas unidirecionais na qual o professor fala e o aluno copia. É nítido que o método antigo no qual só o professor fala é falho. Como acadêmico de Odontologia reconheço que a metodologia ativa não só nos ensina, mas também nos motiva a buscar conhecimento para no futuro ser um profissional mais qualificado”. Estudante 7

"Acredito que as metodologias ativas usadas pela Estomatologia contribuíram sim para o aumento do meu conhecimento, pois com as discussões feitas com meus colegas e as diferentes atividades me fizeram ter mais entusiasmo e vontade de estudar e me aprofundar nos diferentes temas". Estudante 8

\section{CONSIDERAÇÕES FINAIS}

Para disciplinas como Estomatologia, na qual o desenvolvimento de competências como raciocínio lógico, pensamento crítico, interdisciplinaridade, comunicação, atendimento holístico do paciente; são fundamentais para que os resultados de aprendizagem sejam alcançados pelos estudantes de graduação em Odontologia; as metodologias ativas mostram-se ferramentas fundamentais durante todo o processo de ensino e aprendizagem.

Para que os objetivos deste processo sejam plenamente alcançados, destaca-se a importância da capacitação não só de professores como também a orientação aos estudantes, por parte das Instituições de Ensino. Paralelamente, investimentos em salas com estrutura física, de som e imagem, além de materiais específicos são extremamente necessários.

Em contrapartida, há que se considerar outros aspectos relevantes para o sucesso deste processo. Assim, a proporção do número de estudantes por professor torna-se relevante, pois as etapas que fazem parte das atividades envolvendo as metodologias ativas iniciam-se 
sempre fora da sala de aula, seja com o planejamento das atividades, elaboração de materiais ou, muitas vezes, atividades preparatórias extraclasse, as quais necessitam de feedbacks. Em seguida, a outra etapa é realizada presencialmente com os estudantes a qual exige do professor muita atenção e habilidade para o direcionamento da mesma, partindo-se da produção, individual ou em grupo, dos estudantes.

Para que o ensino e aprendizado baseado em competências em Odontologia seja viável, é importante que a matriz curricular do Curso seja estruturada de forma a propiciar este tipo de formação destacando um planejamento partindo de lideranças institucionais com ênfase na responsabilidade profissional dos cirurgiõesdentistas frente ao mercado de trabalho com previsibilidade às necessidades futuras da sociedade, ao invés de um planejamento preocupado apenas com o conteúdo que as disciplinas devem transmitir para os estudantes. Um currículo que permita com prontidão a progressão dos estudantes em diferentes ritmos em relação aos seus colegas, respeitando a individualidade de cada um. Finalmente, um currículo com estrutura horizontal na qual os estudantes progridam, por níveis de habilidades organizadas hierarquicamente e de acordo com o grau de dificuldade, promovendo a aproximação entre o laboratório e a experiência clínica. Neste contexto, a participação ativa dos estudantes de graduação em Odontologia como protagonistas de sua própria formação por meio de metodologias ativas de ensino e aprendizagem é fundamenta ${ }^{15}$.

\section{ABSTRACT \\ Active methodologies used as pedagogic strategy to promote teaching-learning in Dentistry: report of experience}

Traditionally, teaching and learning of Dentistry courses in Brazil have focused on the development of scientific knowledge and technical skills inherent to the profession. However, when considering the demands of today's society, the importance of providing undergraduate Dentistry students with integral, holistic, and competence-based training that goes beyond the profession's technical requirements becomes clear. This article reports the experience of applying active methodologies, including playful activities, as a pedagogic strategy for the promotion of teaching and learning, based on competence-based training, in the Stomatology course at the Dental School of the Pontifícia Universidade Católica do Paraná (PUCPR), Curitiba, Brazil. According to undergraduate students' reports, it was concluded that such active methodologies could be used as a complementary pedagogic strategy for teaching and learning in Dentistry.

Descriptors: Teaching. Learning. Dentistry.

\section{AGRADECIMENTOS}

Este relato de experiência foi resultado da participação em um projeto de formação docente, conduzido pelo CrEARE (Centro de Ensino e Aprendizagem) da Pontifícia Universidade Católica do Paraná, o qual foi promovido e fomentado pela Agência Financiadora de Inovação e Pesquisa (FINEP), entre os anos de 2017 e 2019.

\section{REFERÊNCIAS}

1. Valente, JA. Blended learning e as mudanças no ensino superior: a proposta da sala de aula invertida. Educar 2014; Edição especial (4):79-97.

2. Araújo, IS; Mazur, E. Instrução pelos colegas e ensino sob medida: uma proposta para o engajamento dos alunos no processo de ensino-aprendizagem de Física. Cad Bras Ens Fís. 2013; 30(2):362-84.

3. Morán, J. Mudando a educação com metodologias ativas. 2015 Coleção Mídias Contemporâneas. Convergências Midiá- 
ticas, Educação e Cidadania: aproximações jovens. Vol. II.

4. Prince, M. Does active learning work? A review of research. J Engr Education. 2004; 93(3):223-31.

5. Competencies for the New General Dentist (As approved by the 2008 ADEA House of Delegates). J Dent Educ 2011, 75(7), 932935.

6. Spricigo, CB. Estudo de caso como abordagem de ensino. Pontifícia Universidade Católica do Paraná, 2014. [Acesso em 20 dez. 2018]. Disponível em: https://www.pucpr.br/wp-content/uploads/ 2017/10/estudo-de-caso-como-abordagemde-ensino.pdf

7. Rocha, EF. Metodologias ativas: um desafio além das quatro paredes da sala de aula. 2012. [Acesso em 20 dez. 2018]. Disponível em: http://www.abed.org.br/arquivos/ Metodologias_Ativas_alem_da_sala_de_au la_Enilton_Rocha.pdf

8. Dahms, ML. Problem based learning in engineering education. Pontifícia Universidade Católica do Paraná, 2018. [Acesso em 20 dez. 2018]. Disponível em: https://www.pucpr.br/wp-content/uploads/ 2017/10/artigo-pbl.pdf

9. Almeida, ECS; Venúscolo, DMS; Júnior, WM. A conformação da Odontologia enquanto profissão: uma revisão bibliográfica. RBO. 2002; 59(6):370-3.

10. Saliba, NA; Moimaz, SA; Garbin, CA; Diniz, DG. Dentistry in Brazil: its history and cirrent trends. J Dent Educ. 2009; 73(2):225-31.
11. Scavuzzi, AIF; Gouveia, CVD; Carcereri, DL; Veeck, EB; Ranali, J; Costa, LJ; Morita, MC; Araújo, ME. Rev ABENO. 2015; 15(3):109-13.

12. Queiroz, MG; Dourado, LF. Ensino da Odontologia no Brasil: uma leitura com base nas recomendações e nos encontros internacionais da década de 1960. Histo Ciênc Saúde. Manguinhos. 2009; 16(4):1011-26.

13. Veiga, IPA (Org). Técnicas de ensino: por que não? 21ed. Campinas, SP: Papirus, 2011.

14. Kibble, JD; Bellew, C; Asmar, A; Barkley, L. Team-based learning in large enrollment classes. Adv Physiol Educ. 2016; 40: 43542.

15. William, D; Hendricson, WD; Cohen PA. Future directions in dental school curriculum, teaching, and learning. American Association of Dental Schools. 75th Anniversary Summit Conference, p. 125.

\section{Correspondência para:}

Soraya de Azambuja Berti Couto

e-mail: soraya.berti@pucpr.br

Pontifícia Universidade Católica do Paraná

Escola de Ciências da Vida

Curso de Odontologia

Rua Imaculada Conceição, 1155, Prado Velho

80215-901 Curitiba/PR 